\title{
Efecto del probiótico Saccharomyces boulardii en la prevención de la diarrea asociada con antibióticos en adultos ambulatorios en tratamiento con amoxicilina
}

\author{
María Verónica Bravo ${ }^{1,2}$, Daniel Bunout ${ }^{1}$, Laura Leiva ${ }^{1 a}$, \\ María Pía de la Maza ${ }^{1}$, G ladys Barrera ${ }^{\text {lb, }}$ \\ Javier de la Mazac, Sandra Hirsch ${ }^{1}$. \\ Effect of probiotic Saccharomyces \\ boulardii on prevention of antibiotic- \\ associated diarrhea in adult \\ outpatients with amoxicillin treatment
}

\begin{abstract}
Background: Antibiotic-associated diarrhea is one of the most common adverse effects of antimicrobials. Any antimicrobial can potentially produce diarrhea but beta-lactamics have a higher risk. Among these, amoxicillin is widely indicated in ambulatory practice. One of the alternatives suggested to prevent antibiotic-associated diarrhea, is the use of the probiotic Saccharomyces boulardii. Aim: To evaluate whether the concomitant use of Saccharomyces boulardii and amoxicillin can prevent antibiotic associated diarrhea in ambulatory adults with acute infections diseases, without provoking other adverse effects. Material and Methods: Eighty six adults (aged 15 to 81 years) with acute infectious diseases, excluding those arising in the gastrointestinal tract, that received a prescription of oral amoxicillin for 5 to 10 days, were included. In a controlled randomized, double blind trial, 41 patients were assigned to receive lyophilized Saccharomyces boulardii ( $500 \mathrm{mg} /$ day) during 12 days, and 45 patients were assigned to placebo for the same period. Results: Ten percent of patients (9/86) reported acute diarrhea, 9,8\% (4/41) in the experimental group and $11.1 \%$ $(5 / 45)$ in the control group $(p=100)$. No adverse effects were associated to the use of the probiotic. Conclusions: Saccharomyces boulardii (500 mg/day) did not prevent diarrhea related to amoxicillin (Rev Méd Chile 2008; 136: 981-8).

(Key words: Amoxicillin; Diarrhea; Saccharomyces)

\footnotetext{
Recibido el 23 de octubre, 2007. Aceptado el 11 de abril, 2008.

Financiamiento: Laboratorio TOSCANA.

${ }^{1}$ Instituto de Nutrición y Tecnología de los Alimentos, Universidad de Chile. Santiago de Chile. ${ }^{2}$ Hospital Dr. Sótero del Río.

aTecnólogo médico. ${ }^{\mathrm{b}}$ Enfermera. ${ }^{\mathrm{c}}$ Alumno de Medicina, Universidad de Chile
}

Correspondencia a: Dra. María Verónica Bravo. Servicio de

Medicina, Hospital Dr. Sótero del Río, Santiago, Chile.

Fono: 56-2-3536604. Fax: 56-2-2955026.

E mail: vbravol@vtr.net 
L diarrea asociada con antibióticos (DAA) se define como un cuadro diarreico inexplicado que ocurre en relación a la administración de antibióticos ${ }^{1}$, en una persona sin patología gastrointestinal previa. Los antibióticos podrían producir cambios significativos en la composición de la microflora intestinal de intensidad y duración variables ${ }^{2}$. Estos cambios serían el resultado de su acción sobre la mucosa intestinal, la directa supresión de la flora entérica habitual, alteraciones de su función normal y disponibilidad de nutrientes ${ }^{3-6}$. En algunos antibióticos, el mecanismo sería por cambios en la motilidad intestinal. La DAA puede aparecer desde pocas horas de iniciado el tratamiento antibiótico hasta dos a seis semanas de terminado el tratamiento antibiótico ${ }^{4}$, no obstante, la máxima incidencia de DAA es durante las primeras dos a tres semanas de iniciado el tratamiento antibiótico ${ }^{8,12}$. La aparición de DAA depende del antibiótico utilizado. Los antibióticos que se asocian con mayor frecuencia a DAA son los beta-lactámicos, cefalosporinas y la clindamicina, pero en la práctica cualquier antibiótico puede inducirla ${ }^{1,4,13}$.

La frecuencia de DAA, tanto en pacientes hospitalizados como ambulatorios, varía entre $5 \%$ y $30 \% 1,7,8,10,-13$. La ocurrencia de DAA también puede depender de factores relacionados con el huésped, como son la edad, las co-morbilidades de los pacientes y la prolongación de la hospitalización ${ }^{4,14}$.

Diversos estudios han usado los probióticos para prevenir o tratar la DAA. Los probióticos se definen como organismos vivos y viables, que pueden ser exógenos al individuo, no deben ser toxigénicos ni enteropatógenos, deben ser capaces de sobrevivir y colonizar el intestino del huésped transitoriamente, deben ser resistentes a la acción del ácido gástrico, la bilis y la destrucción cuando son procesados, pueden ser reconocidos en las deposiciones y aislados fácilmente de la flora indígena y deben ser capaces de permanecer viables durante su uso y almacena$\mathrm{je}^{15,16}$

Se ha planteado que en humanos el mecanismo protector de los probióticos se debería a la producción de ácidos orgánicos, bacteriocinas y péptidos, que interferinían con la acción de patógenos gastrointestinales y a un efecto estimulador de la inmunidad general ${ }^{17,18}$.
Uno de los probióticos más frecuentemente usados en clínica es Saccharomyces boulardii, tanto en la prevención ${ }^{10,19-21}$ como en el tratamiento de $\mathrm{DAA}^{9}$, con resultados contradictorios. Saccharomyces boulardii dado a hámsteres en forma profiláctica, disminuiría la mortalidad inducida por clindamicina ${ }^{22}$. Estudios in vivo e in vitro muestran que Saccharomyces boulardii posee la capacidad de inhibir el efecto de bacterias patógenas, y no es destruido por antibióticos ${ }^{23}$. Produce una proteasa de $54 \mathrm{kDa}$ que destruiría tanto la toxina A del Clostridium difficile, como también el receptor intestinal que une ambas toxinas A y B $\mathrm{B}^{24}$.

Nuestro objetivo fue evaluar si el uso de Saccharomyces boulardii ejerce un efecto preventivo en la aparición de DAA asociada al uso de amoxicilina, que es el antibiótico más ampliamente usado en adultos ambulatorios en el tratamiento de infecciones agudas, principalmente respiratorias.

\section{Pacientes y Métodos}

Pacientes. Se preseleccionaron pacientes de 15 años o más, que asistieron a un consultorio dependiente del Servicio de Salud Metropolitano Oriente (SSMO), entre 2004 y 2005, a quienes se indicó amoxicilina oral por 5 a 10 días. Se consideraron elegibles aquellos pacientes con el diagnóstico de una infección aguda, que no fuera del tracto digestivo, con indicación de usar amoxicilina dentro de las 24 horas previas del ingreso al estudio, que no hubiesen usado antibióticos ni presentado diarrea en los últimos 30 días, y que accedieran a participar voluntariamente, firmando un consentimiento informado.

Se excluyeron personas embarazadas, con enfermedades crónicas avanzadas (insuficiencia renal terminal en diálisis crónica, daño hepático crónico con ascitis, diarrea crónica, cáncer avanzado o SIDA), portadores de válvulas protésicas cardíacas, sujetos en tratamiento con terapia inmunosupresora, quimioterapia o radioterapia, que estuvieran tomando lactulosa u otros catárticos, antidepresivos (inhibidores de la MAO) o que consumieran probióticos alimentarios en forma habitual (yogurt "casero", etc.) al momento de ingresar al estudio. 
Métodos. Se realizó un estudio prospectivo aleatorio, de doble control, ciego. Se formaron dos grupos en forma aleatoria, un grupo (GS), que recibió amoxicilina y Saccharomyces boulardii liofilizado (levadura seca de Saccharomyces boulardii $250 \mathrm{mg}$, un mínimo de 5,1 x $10^{9}$ células viables en cada cápsula), 1 cápsula v.o. cada 12 horas, equivalente a $500 \mathrm{mg} / \mathrm{d}$, durante 12 días, y un grupo control (GC), que recibió amoxicilina y placebo (lactosa micronizada), 1 cápsula v.o. cada $12 \mathrm{~h}$, durante 12 días. Cada uno recibió al azar un frasco codificado y sellado con placebo o probiótico, indistinguibles físicamente uno del otro. Desde ese día, se realizó seguimiento telefónico diario por 3 semanas, al paciente o familiar cercano, consultando sobre la adherencia al protocolo, hábito intestinal, consistencia de deposiciones, presencia 0 ausencia de diarrea y síntomas gastrointestinales asociados, como náuseas, vómitos, anorexia, intolerancia oral, distensión abdominal, aerofagia 0 meteorismo, compromiso del estado general, suspensión del régimen oral, nueva consulta médica, $\mathrm{y}$ cualquier síntoma nuevo que relatara el paciente y que subjetivamente lo atribuyera al tratamiento.

Cada paciente se controló en el policlínico entre el $5^{\circ}$ y el $7^{\circ}$ día, para evaluar adherencia al tratamiento mediante el control de número de cápsulas consumidas.

En aquellos pacientes que presentaron diarrea, se solicitó, coprocultivo corriente, leucocitos fecales y detección de toxina A para Clostridium difficile en deposiciones. Posteriormente se inició terapia, según el estado clínico del paciente y su evolución por el equipo del protocolo.

Se definió DAA como el cambio de hábito intestinal con al menos tres deposiciones acuosas, blandas o disgregadas en el día, durante al menos dos días consecutivos y hasta la $3^{a}$ a semana de iniciado el tratamiento antibiótico, percibidas por el paciente como anormales $8,10,12,19$. La duración de la diarrea se definió como el número de días totales con diarrea, incluidos los 2 días consecutivos iniciales ${ }^{12}$, durante las 3 semanas de observación.

La frecuencia intestinal habitual se estimó como el cuociente entre el número de deposiciones y el intervalo, en días, informada en la anamnesis por cada sujeto. La frecuencia durante el estudio se calculó como el promedio entre el número de deposiciones y el número de días efectivamente observados.

Este estudio contó con la aprobación del Comité de Ética del INTA, Universidad de Chile.

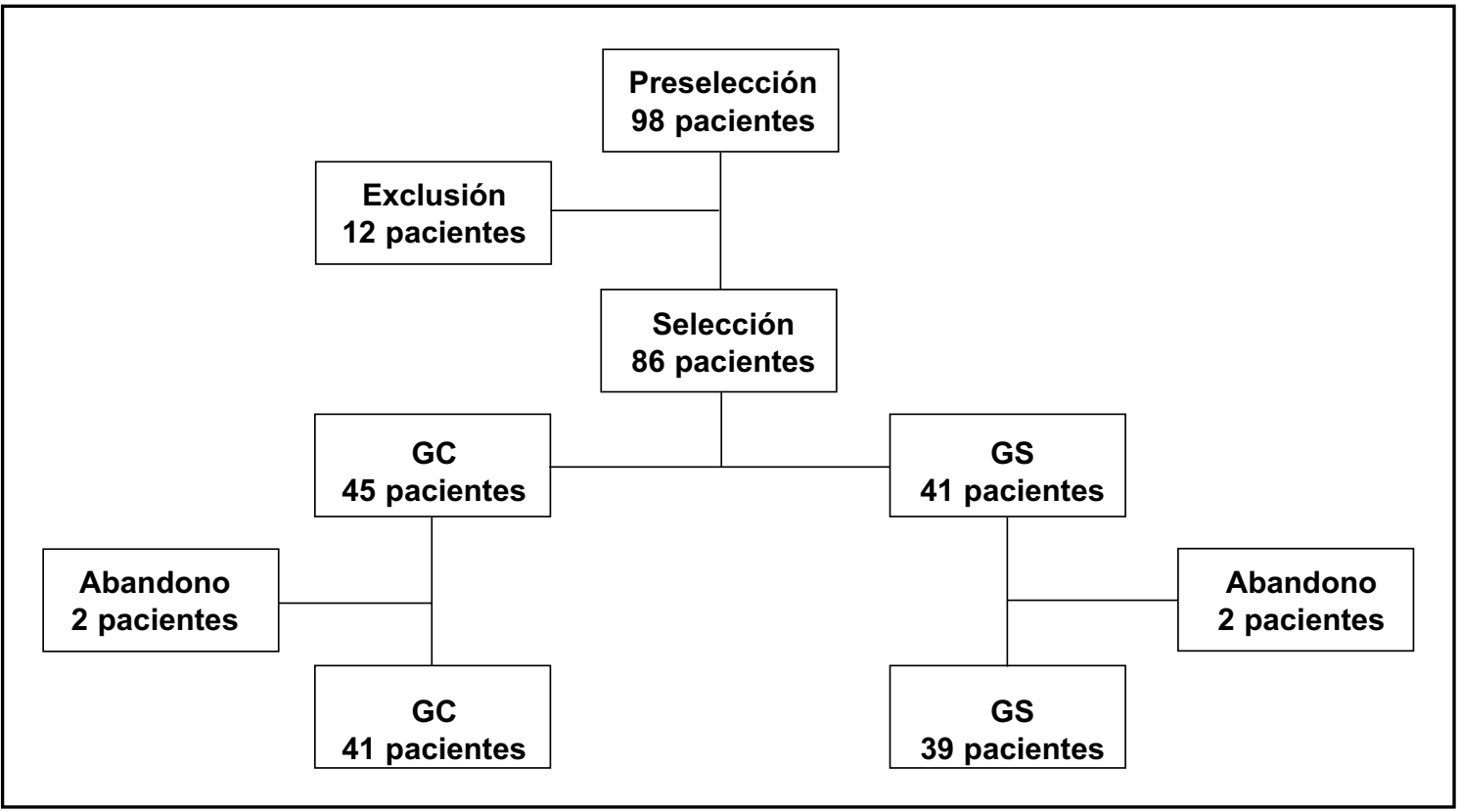

Figura 1. Descripción de los grupos del estudio. GC: Grupo Control. GS: Grupo Saccharomyces. 
Tabla 1. C aracterísticas basales de la población del estudio

\begin{tabular}{|c|c|c|}
\hline Pacientes & $\begin{array}{c}\text { Grupo C } \\
\mathrm{n}=45\end{array}$ & $\begin{array}{c}\text { Grupo S } \\
\mathrm{n}=41\end{array}$ \\
\hline Hombres [n (\%)] & $9(20)$ & $11(27)$ \\
\hline Mujeres [n (\%)] & $36(80)$ & $30(73)$ \\
\hline $\begin{array}{l}\text { Edad [años }(\text { Mean } \pm \text { SD })] \\
\text { (rango) }\end{array}$ & $\begin{array}{c}50,98 \pm 17,94 \\
\quad(15 \text { a } 81)\end{array}$ & $\begin{array}{c}49,78 \pm 20,50 \\
(15 \text { a } 79)\end{array}$ \\
\hline $\begin{array}{l}\text { Frecuencia usual de hábito intestinal } \\
\text { - }<1 \text { vez / día [n (\%) } \\
\text { - } 1 \text { vez / día [n (\%)] } \\
\text { - } 2 \text { veces / día [n (\%)] } \\
\text { - } \geq 3 \text { veces / día [n (\%)] }\end{array}$ & $\begin{array}{c}22(48,89) \\
19(42,22) \\
4(8,89) \\
0(0)\end{array}$ & $\begin{array}{c}12(29,27) \\
19(46,34) \\
9(21,95) \\
1(2,44)\end{array}$ \\
\hline $\begin{array}{l}\text { Antecedentes mórbidos [n (\%)] } \\
\text { - Cardiovasculares [n (\%)] } \\
\text { - Diabetes Mellitus [n (\%)] } \\
\text { - Intestino irritable [n (\%)] } \\
\text { - Otras [n (\%)] }\end{array}$ & $\begin{aligned} 31 & (68,89) \\
17 & (37,78) \\
4 & (8,89) \\
3 & (6,67) \\
22 & (48,89)\end{aligned}$ & $\begin{array}{l}30(73,17) \\
14(34,15) \\
6(14,63) \\
4(9,76) \\
18(43,90)\end{array}$ \\
\hline $\begin{array}{l}\text { Infección aguda } \\
\text { - } \text { Respiratoria [n (\%)] } \\
\text { - } \text { Dental [n (\%)] }\end{array}$ & $\begin{array}{c}37(82,22) \\
8(17,78)\end{array}$ & $\begin{array}{l}39(95,12) \\
2(4,88)\end{array}$ \\
\hline
\end{tabular}

$*_{p}<0.05=$ significativo

Estadística. El tamaño muestral estimado se basó en un riesgo basal de DAA por amoxicilina de $30 \%$, con una reducción de riesgo de $50 \%{ }^{25}$, se definió un nivel de confianza del 95\%, (error a de $5 \%$ y error b de 20\%). El ciego se mantuvo durante todo el protocolo para los pacientes, médico tratante y hasta después del análisis de los datos. Se analizó por intención de tratar y efectividad.

Se usó estadística descriptiva incluyendo promedios, desviación estándar, y porcentajes. Para variables continuas se usó test t de Student y para variables nominales, test de Chi cuadrado o test exacto de Fisher con una probabilidad de $<0,05$ considerada estadísticamente significativa.

\section{Resultados}

Se incluyeron 86 pacientes, de 98 pacientes preseleccionados que aceptaron ingresar al estudio, 45 en el GC y 41 en el GS (Figura 1). Doce pacientes fueron excluidos del estudio debido a que cinco estaban tomando antibióticos más allá de $48 \mathrm{~h}$, dos presentaban diarrea crónica, dos tenían dificultad de contacto telefónico, uno tomó amoxicilina-clavulánico, uno presentó diarrea el primer día, y uno por temor a intolerancia se negó a participar.

Los pacientes del GC y GS fueron similares en sus características basales, (Tabla 1), al analizar los pacientes que efectivamente iniciaron el tratamiento, las características se mantienen (Tabla 2).

Dos pacientes en cada grupo no tomaron el probiótico o el placebo. En el GC, un paciente se hospitalizó por cuadro de abdomen agudo y otro paciente viajó fuera de la ciudad. En el GS dos pacientes no tomaron amoxicilina ni probiótico, uno porque mejoró su sintomatología respiratoria y otro para evitar molestias asociadas a la ingesta de antibióticos. Los cuatro pacientes se excluyeron en el análisis de efectividad. Los días de seguimiento promedio fueron $20,14 \pm 3$,56 días en el GC y de 19,38 $\pm 3,42$ días en el GS (95\% IC: 0,78 a 2,30, $p=0,328$ ). Ambos grupos fueron similares en cuanto a la duración y cantidad de 
Tabla 2. Resumen de frecuencia de diarrea y estudio en los pacientes que
efectivamente participaron del estudio

\begin{tabular}{|c|c|c|}
\hline Diarrea & $\begin{array}{c}\text { Grupo C } \\
\mathrm{n}=43\end{array}$ & $\begin{array}{c}\text { Grupo S } \\
\mathrm{n}=39\end{array}$ \\
\hline № de pacientes (\%) & $5(11,63)$ & $3(7,69)$ \\
\hline № de días (promedio \pm DS) & $4,2 \pm 1,3$ & $3,5 \pm 1,73$ \\
\hline \multicolumn{3}{|l|}{ Amoxicilina } \\
\hline № de días (promedio \pm DS) & $7,47 \pm 1,56$ & $7,26 \pm 1,57$ \\
\hline cantidad (promedio \pm DS) & $21,53 \pm 4,81$ & $21,28 \pm 5,03$ \\
\hline \multicolumn{3}{|l|}{ Probiótico / placebo } \\
\hline $\mathrm{n}^{\mathrm{o}}$ de días (promedio $\pm \mathrm{DS}$ ) & $11,49 \pm 2,24$ & $11,41 \pm 2,05$ \\
\hline cantidad (promedio \pm DS) & $23,00 \pm 4,48$ & $22,72 \pm 4,08$ \\
\hline \multicolumn{3}{|l|}{ Hábito intestinal } \\
\hline Frecuencia en el estudio (promedio \pm DS) & $1,16 \pm 0,41$ & $1,16 \pm 0,41$ \\
\hline
\end{tabular}

$*_{p}<0,05=$ significativo

unidades tomadas de amoxicilina, Saccharomyces boulardii y placebo (Tabla 2 ).

Presentó DAA el 9,3\% pacientes (8/86) del total de la muestra. En el GC fue de 11,1\% (5/45) y $7,3 \%(3 / 41)$ en el GS. Para los pacientes que efectivamente se trataron, la frecuencia fue similar con $11,6 \%(5 / 43)$ del GC, y $7,7 \%(3 / 39)$ del GS (Tabla 2). La duración de la diarrea como también la frecuencia del hábito intestinal durante el período observado, fue similar en ambos grupos (Tabla 2).

De los ocho pacientes que presentaron diarrea, sólo cuatro se realizaron exámenes, cuatro pacientes no asistieron al control, debido a la escasa repercusión sistémica percibida por ellos. Las muestras de deposición se procesaron dentro de las 2 horas de emitidas. Tanto el coprocultivo corriente como la toxina A para $\mathrm{C}$ difficile, fueron negativos, y 3 de 4 muestras de leucocitos fecales fueron positivas, dos en el GC y una en el GS.

Los síntomas más frecuentes encontrados en ambos grupos al usar probiótico o placebo, con amoxilina, fueron los gastrointestinales tales como, dispepsia, aerofagia, pirosis y dolor abdominal, la frecuencia fue similar $64,4 \%(29 / 45)$ en el GC y en $65,9 \%(27 / 41)$ en el GS ( $p=0,913)$. No se evaluó la frecuencia basal de estos síntomas, previa al uso de amoxicilina. Cuatro pacientes en el grupo GC y tres en el grupo GS relataron efectos adversos. De estos, dos pacientes en cada grupo relataron distensión o dolor abdominal.

\section{DisCUSIÓN}

En este estudio se observó una incidencia de DAA menor a la esperada.

Existen escasos estudios que evalúan la DAA en adultos ambulatorios, pero según publicaciones recientes ${ }^{12,25-27}$, su incidencia varía ampliamente y depende del tipo de antibiótico usado. En pacientes ambulatorios mayores de 18 años, con sinusitis aguda bacteriana, tratados con gatifloxacino 0 amoxicilina-clavulánico durante 10 días, 7 y $14 \%$ presentaron diarrea respectivamente durante los 28 días de observación ${ }^{26}$. En mayores de 12 años con infecciones de piel y tejidos blandos, tratados con cefalosporinas por 10 días, presentaron diarrea $7 \%$ de los que tomaron cefuroximo, $8 \%$ de los que tomaron cefadroxilo y con dosis bajas y altas de cefditoren presentaron diarrea $14 \%$ y $19 \%$, respectivamente ${ }^{27}$. En mayores de 18 años con infecciones respiratorias altas que tomaron entre 4 y 14 días distintos tipos de antibióticos, el 18\% aproximadamente presentó diarrea, durante 14 días de observación ${ }^{12}$. En pacientes con artritis inflamatoria crónica tratados con ceftriaxona por 2 semanas, $48 \%$ presentó diarrea ${ }^{28}$. 
Amoxicilina es uno de los antibióticos orales más usados en adultos ambulatorios. La literatura informa diversas frecuencias de efectos adversos relacionados al uso de este antibiótico. En pacientes pediátricos ambulatorios con otitis media aguda en tratamiento con amoxicilina o azitromicina durante 10 días $^{30}$, la incidencia de efectos adversos fue de $20 \%$ y $29 \%$, respectivamente, siendo la diarrea el más frecuente y mayor en el grupo con amoxicilina (17,5\% y $8,2 \%$ respectivamente, $\mathrm{p}=0,017)$. En mayores de 12 años con rinitis aguda purulenta, De Sutter reportó diarrea como principal efecto adverso en $29 \%$ de 170 pacientes del grupo que tomó amoxicilina por 10 días, versus 19\% de 164 pacientes en el grupo con placebo, con un riesgo absoluto de 10\% para el primer grupo 25 , sin diferencias significativas en otros síntomas. Petitptretz ${ }^{29}$, en pacientes que tomaron $3 \mathrm{~g} /$ día de amoxicilina durante 10 días, describe efectos adversos en el 20,2\% (42/208), siendo el más frecuente alteración de las pruebas hepáticas y elevación aislada de GGT (7,2\% y $4,3 \%)$. Sólo 3,8\% (8/208) de los pacientes presentó diarrea. Se encontraron náuseas y vómitos en $0,5 \%$ respectivamente. En nuestro estudio, los eventos gastrointestinales como náuseas y dolor abdominal fueron más frecuentes.

Encontramos un riesgo basal de diarrea en el grupo que recibió amoxicilina de 11,1\% (5/45) en el grupo control, que es menor al encontrado por De Sutter, pero si se considera la diferencia de riesgo de $10 \%$, este porcentaje sería similar al encontrado en nuestro estudio y es mayor que el reportado por Petitptretz. La menor frecuencia de diarrea observada en el grupo control, comparada con nuestra estimación inicial para calcular el tamaño muestral, es una limitación de este estudio, debido a que se habría necesitado que ningún paciente del grupo intervenido hubiera tenido diarrea para que la diferencia hubiera sido significativa. Pero la significación estadística de la diferencia en frecuencia de diarrea observada entre grupos (11\% y 7\%), no habría mejorado al aumentar el tamaño muestral, incluso a 200 pacientes por grupo.

En relación al uso preventivo de Saccharomyces boulardi en DAA, existen pocos estudios que evalúan su efecto preventivo, administrado concomitantemente con antibióticos, en pacientes con infecciones agudas. Sólo se pueden comparar estudios que han utilizado distintos tipos y duración de antibióticos y distintas dosis y duración de S. boulardii. No conocemos estudios que evalúen el uso conjuntamente de amoxicilina y S. boulardii.

En pacientes hospitalizados, Surawicz ${ }^{19}$ y $\mathrm{M}^{\mathrm{C} F a r l a n d}{ }^{10}$, encontraron una frecuencia de diarrea de $9,5 \%$ vs $21,8 \%$ y $7,2 \%$ vs $14,6 \%$, respectivamente, en pacientes que recibieron $\mathrm{S}$. boulardii $1 \mathrm{~g} /$ día, al compararlos con placebo, con una reducción del riesgo relativo de 57\% (95\% IC: 4 a 100) y 51\% (95\% IC: -9 a 100). Sin embargo, sólo en el primer trabajo la diferencia es significativa. En sus respectivos metanálisis, D'Souza y Cremonini ${ }^{31,32}$ sugieren que los probióticos pueden prevenir la diarrea si se asocian con antibióticos desde el inicio del tratamiento. En estos estudios, el riesgo de diarrea disminuyó en $39 \%$ y $40 \%$, respectivamente con el uso de probióticos. En el análisis de D'Souza, el beneficio sería similar para S. boulardii y para otros probióticos, pero el tipo y número de antibióticos y la duración del seguimiento variaban ampliamente en cada estudio original. En 3 de 4 trabajos que utilizaron S. boulardii no se consiguieron beneficios claros con este probiótico, lo que se atribuyó a diferencias en dosis y duración del tratamiento como también a variaciones en el seguimiento.

Las dosis de S. boulardii administradas en nuestro estudio estuvieron dentro del rango utilizado en otros trabajos (226 mg y $1 \mathrm{~g})^{10,19,20}$. No se usaron dosis mayores debido a los potenciales efectos adversos descritos en la literatura, como sepsis o endocarditis infecciosa. Estos efectos se han presentado con dosis usualmente mayores de $500 \mathrm{mg} /$ día y en pacientes con compromiso de la inmunidad, cáncer activo y procesos patológicos con mayor riesgo de alteración de la permeabilidad intestinal ${ }^{33}$.

No existen diferencias en eventos adversos, que tengan expresión clínica significativa dentro de cada grupo, como tampoco entre ambos grupos, antes y durante el período observado.

En conclusión, en pacientes ambulatorios con infecciones agudas respiratorias altas y dentales tratados con amoxicilina 5-10 días, la administración concomitante de Sachoromyces boulardii (500 mg/día) no previno la diarrea asociada a este antibiótico. 
En la población de adultos ambulatorios estudiada, no se encontraron efectos adversos especialmente atribuidos al uso de este probiótico.

Agradecemos a la Dras. Sylvia Echavami e Isabel Hoffmann por sus ideas y apoyo, al personal del Consultorio

\section{REFERENCIAS}

1. Bartiett JG. Antibiotic-associated diarrhea. N Engl J Med 2002; 346: 334-40.

2. De La Cochetiere MF, Durand T, Lepage P, Bourreilie A, Galmiche JP, Dore J. Resilience of the Dominant Human Fecal Microbiota upon Short-Course Antibiotic Challenge. J Clin Microbiol 2005; 43: 558892.

3. LEVY J. The Effects of Antibiotic Use on Gastrointestinal Function. Am J Gastroenterol 2000; 95 (suppl): S8-S10.

4. Bergogne-Bérézin E. Treatment and Prevention of Antibiotic-Associated Diarrhea. Int J Antimicr Ag 2000; 16: 521-6.

5. Young VB, Schmidt TM. Antibiotic-Associated Diarrhea Accompanied by Large-Scale Alterations in the Composition of the Fecal Microbiota. J Clin Microbiol 2004; 42: 1203-6.

6. Caron F, Ducrotte P, Lerebours E, Colin R, Humbert G, DenIS P. Effects of Amoxicillin-Clavulanate Combination on the Motility of the Small Intestine in Human Beings. Antimicrob Agents Chemother 1991; 35: 1085-8.

7. THOMAs MR, LUTIN SC, O SMOND DR, CoRR AP, Weaver AL, LOHSE CM. Lack of Effect of Lactobacillus GG on Antibiotic-Associated Diarrhea: A randomized, Placebo-Controlled Trial. Mayo Clin Proc 2001; 76: 883-9.

8. Armuzzi A, Cremonini F, Bartolozzi F, Canducci F, Candeli M, OJetti V et al. The effect of oral administration of Lactobacillus GG on antibioticassociated gastrointestinal side-effects during Helicobacter pylori eradication therapy. Aliment Pharmacol Ther 2001; 15: 163-9.

9. McFariand LV, Surawicz C, Greenberg RN, Feketty R, ELMER GW, Moyer KA et aL. Randomized PlaceboControlled Trial of Saccharomyces boulardii in Combination with Standard Antibiotics for Clostridium difficile Disease. JAMA 1994; 271: 1913-8.

10. McFariand LV, Surawicz C, Greenberg RN, Elmer GW, Moyer KA, Melcher SA et al. Prevention of ß-Lactamassociated diarrhea by Saccharomyces boulardii compared with placebo. Am J Gastroenterol 1995; 90: 439-48.
Félix de Amesti del SSMO, por su colaboración, especialmente a su Directora y el personal del SOME que nos facilitó los listados diarios de pacientes, como también a Nancy Cruz Cabrera, técnico del laboratorio del INTA, por su dedicación al proyecto, María Valenzuela Madrid, secretaria del laboratorio y al personal del CEDINTA.

11. Högenauer C, Hammer HF, Krejs G, Retsinger EC. Mechanisms and Management of Antibiotic-Associated Diarrhea. Clin Infect Dis 1998; 27: 702-10.

12. Beaugerie L, Flahault A, Barbur F, Atlans P, Lalande V, Cousin P, M Cadilhac, \& Petit JC (for The Study GrouP). Antibiotic-associated diarrhoea and Clostridium difficile in the community. Aliment Pharmacol Ther 2003; 17: 905-12.

13. Wiström J, Norrby SR, Myhre EB, Eriksson S, GransTRÖM G, LAGERGREN L ET aL. Frequency of antibioticassociated diarrhoea in 2462 antibiotic-treated hospitalized patients: a prospective study. J Antimicrob Chemoter 2001; 47: 43-50.

14. Alfa MJ, Du T, Beda G. Survey of Incidence of Clostridium difficile Infection in Canadian Hospitals and Diagnostic Approaches. J Antimicrob Chemoter 1998; 36: 2076-80.

15. CoLins MD, GiBson GR. Probiotics, prebiotics, and synbiotics: appraches for modulating the microbial ecology of the gut. Am J Clin Nutr 1999; 69 (suppl): 1052S-7S.

16. Bengmark S. Bioecologic Control of the Gastrointestinal Tract: The Role of Flora and Supplemented Probiotics and Synbiotics. Gastroenterol Clin N Am 2005; 34: 413-36.

17. De Roos NM, Katan MB. Effects of probiotic bacteria on diarrhea, lipid metabolism, and carcinogenesis: a review of papers published between 1988 and 1998. Am J Clin Nutr 2000; 71: 405-11.

18. Gill HS, Rutherford KJ, Cross ML, Gopal PK. Enhancement of immunity in the elderly by dietary supplementation with the probiotic Bifidobacterium lactis HN019. Am J Clin Nutr 2001; 74: 833-9.

19. Surawicz CM, Elmer GW, Speelman P, MC Farland LV, ChinN J, VAN Bele G. Prevention of AntibioticAssociated Diarrhea by Saccharomyces boulardii: A prospective Study. Gastroenterology 1989; 96: 981-8.

20. Lewis SJ, PotTs LF, BarRY RE. The Lack of therapeutic effect of Saccharomyces boulardii in the prevention of antibiotic-related diarrhoea in elderly patients. J Infect 1998; 36: 171-4.

21. Kotowska M, Albrecht P, Szajewska H. Saccharomyces boulardii in the prevention of antibiotic-associated diarroea in children: a randomized double-blind 
placebo-controlled trial. Aliment Pharmacol Ther 2005; 21: 583-90.

22. Toothaker RD, Elmer GW. Prevention of Clindamycin-Induced Mortality in Hamsters by Saccharomyces boulardii. Antimicrob Agents Chemother 1984; 26: 552-6.

23. Buts JP, Bernasconi P. Saccharomyces boulardii: Basic Science and Clinical Applications in Gastroenterology. Gastroenterol Clin N Am 2005; 34: 515-32.

24. Castagluuolo I, Riegler MF, Valenick L, Thomas-Lamont J, Ротнoulakis $\mathrm{CH}$. Saccharomyces boulardii Protease Inhibits the Effects of Clostridium difficile Toxins $\mathrm{A}$ and $\mathrm{B}$ in Human Colonic Mucosa. Infect Immun 1999; 67: 302-7.

25. De Sutter AI, De Meyere MJ, Chritiaens TC, Van Driel ML, Peersman W, De Maeseneer JM. Does amoxicillin improve outcomes in patients with purulent rhinorrhea? A pramatic randomized double-blind controlled trial in family practice. J Fam Pract 2002; 51: 317-23.

26. Sher LD, McAdoo MA, Bettis RB, Turner MA, Li NF, Pierce PF. A Multicenter, Randomized, InvestigatorBlinded Study of 5-10 day Gatifloxacin versus 10day Amoxicillin/clavulanate in Patients with Acute Bacterial Sinusitis. Clin Ther 2002; 24: 269-81.

27. Bucko AD, Hunt BJ, Kidd SL, Hom R. Randomized, Double-Blind, Multicenter Comparison of Oral Cefditoren 200 or $400 \mathrm{mg}$ BID with either Cefuroxime
$250 \mathrm{mg}$ in Uncomplicated Skin and Skin-.Structure Infections. Clin Ther 2002; 24: 1134-47.

28. Caperton EM, Heim-Duthoy KL, Matzke GR, Peterson PK, Johnson RC. Ceftriaxone Therapy of Chronic Inflammatory Arthritis. A Double-Blind Placebo Controlled Trial. Arch Intern Med 1990; 150: 1677-82.

29. Petitpretz P, Arvis P, Marel M, Moita J, Urueta J, the CAP5 Moxifloxacin Study Group. Oral Moxifloxacin vs High-Dosage Amoxicillin in the Treatment of Mild-to-Moderate, Community-Acquired, Suspected Pneumococcal Pneumonia in Adults. CHEST 2001; 119: 185-95.

30. Arguedas A, Emparanza P, Schwartz RH, Soley C, Guevara S, De Capraris PJ ET aL. A randomized, multicenter, double blind, double dummy trial of single dose azithromycin versus high dose amoxicillin for treatment of uncomplicated acute otitis media. Pediatr Infect Dis J 2005; 24: 153-61.

31. D’Souza A, Rajkumar CH, Cooke J, Bulpitt CJ. Probiotics in prevention of antibiotic associated diarrhoea: neta-analysis. BMJ 2002; 324: 1361-6.

32. Cremonini F, Di Caro S, Nista EC, Bartolozzi F, Capelu G, GASBARRINI G \& GASBARRINI A. Meta-analysis: the effect of probiotic administration on antibiotic associated diarrhoea. Aliment Pharmacol Ther 2002; 16: 1461-7.

33. Boyle RJ, Robins-Browne RM, Tang MLK. Probiotic use in clinical practice: what are the risks? Am J Clin Nutr 2006; 83: 1256-64. 